\title{
Convivencia escolar mediante la investigación como estrategia pedagógica ${ }^{1}$
}

\section{School coexistence through research as a pedagogical strategy}

DOI: http://dx.doi.org/10.17981/cultedusoc.9.1.2018.07

Fecha de recepción: 07/04/2018. Fecha de aceptación: 05/07/2018

Luz Calderón-Benítez ${ }^{2}$ (D)

Lizeth Henry-Pérez; Ana Castillo-Camargo; María López-Fontalvo;

Gladys Quiroga-Luque; Clara Uribe-Torres; Ubaldina Contreras-González

IED San José de Kennedy, sede principal (Colombia)

licluz81@gmail.com

Para citar este artículo

Calderón-Benítez, L., Henry-Pérez, L., Castillo-Camargo, A., López-Fontalvo, M., Quiroga-Luque, G., Uribe-Torres, C. y ContrerasGonzález, U. (2018). Convivencia escolar mediante la investigación como estrategia pedagógica. Cultura. Educación y Sociedad 9(1), 95-105. DOI: http://dx.doi.org/10.17981/cultedusoc.9.1.2018.07

\section{Resumen}

La convivencia sana de los integrantes de una comunidad educativa, es uno de los temas más importantes a desarrollar debido a que influyen en el desarrollo integral de los estudiantes. El estudio tiene como objetivo, mejorar la convivencia dentro de la institución educativa San José entre los estudiantes a través del empleo de la investigación como una estrategia pedagógica. Se llevó a cabo bajo una metodología de tipo cualitativo, desarrollada desde la investigación como estrategia pedagógica (IEP) donde se utilizó la técnica de observación directa, el estudio contó con la participación de ochenta (80) estudiantes distribuidos en los grados de sexto $\left(6^{\circ}\right)$ a noveno $\left(9^{\circ}\right)$ de la Institución Educativa Departamental San José, sede principal, ubicada en el municipio de Pueblo viejo Magdalena. Finalmente se observó que la convivencia de los estudiantes mejoró considerablemente gracias al uso de la investigación como estrategia de aprendizaje y desarrollo, demostrando que los estudiantes interiorizaron valores humanos que permitieron disminuir situaciones problemáticas en su vida escolar cotidiana.

Palabras clave: convivencia escolar, investigación, estrategia pedagógica.

\begin{abstract}
The peaceful coexistence of the members of an educational community is one of the most important issues to develop, because they influence the overall development of the students. The objective of the study is to improve coexistence within the educational campuses among students through the use of tactics that ensure positive results. Methodologically oriented under a qualitative orientation, using the methodological load of research as a pedagogical strategy (IEP), the study had the participation of eighty (80) students distributed in grades six (6) to ninth $\left(9^{\circ}\right)$ of the Departmental Educational Institution San José, headquarters, located in the municipality of Pueblo Viejo Magdalena, where techniques such as interviews and participant observation were used. Finally, it was observed that the coexistence of students improved considerably thanks to the use of research as a learning and development strategy.
\end{abstract}

Keywords: school coexistence, research, pedagogical strategy.

\footnotetext{
${ }^{1}$ Este artículo ha sido derivado del Programa de Fortalecimiento de la Cultura Ciudadana y Democrática CT+I a través de la IEP apoyada en TIC en el Departamento de Magdalena: CICLÓN. Desarrollado con docentes miembros del Grupo de investigación "Guardianes de San José" pertenecientes a la Institución Educativa Departamental San José de Kennedy, sede principal.

${ }^{2}$ Líder del grupo de investigación los "Guardianes de San José".
} 


\section{Introducción}

Es importante abordar la convivencia en las instituciones educativa, pues estas marcaran las relaciones a futuro, por tal motivo se aborda este tema que tiene como objetivo fortalecer la convivencia escolar mediante la investigación como estrategia pedagógica en la escuela. La convivencia constituye uno de los aspectos más importantes en las relaciones humanas como manifestación natural de esta, se puede encontrar en ocasiones cierto tipo de desacuerdos, comunicación inadecuada, etc., que puede dar lugar a conflictos entre personas, por tanto, la convivencia y el conflicto se presentan en un mismo escenario, pero no podemos dejar de lado la idea de que vivimos en una sociedad donde en muchas ocasiones se quiere resolver cualquier tipo de conflicto de manera violenta y agresiva; la violencia crea expectación y no es extraño que a veces aparezca resaltada de manera no muy rigurosa (Grande, 2010).

Se ha escrito mucho acerca de la forma como solucionar las diferentes problemáticas en el aula y en las instituciones educativas, pero nunca es suficiente hasta que un proyecto o técnica implementada logre de una vez por todas aclimatarnos en la atmosfera de la paz y la sana convivencia. Convivir supone desarrollar la vida en compañía de otros; así mismo, supone que la armonía existente en ella busque un espacio compartido en el que se perciba un desarrollo que no limite a los demás. Desde un principio, se convive con el núcleo familiar y, a medida que se va creciendo, se integran nuevos grupos, los cuales desarrollan otro tipo de actividades en escenarios diferentes, como es el caso del colegio, campo que a su vez se presta tanto para buenas como malas relaciones interpersonales (Ocampo, Briceño, Hernández \& Olano, 2010).

Por su parte López (2003) argumenta que el tiempo y el espacio en las redes generan formas distintas de comunicación, que deben ser muy bien estudiadas desde la intervención pedagógica, ya que las redes sociales, propician mayor desarrollo de la libertad de expresión (Valenzuela \& Rochin, 2013) y tanto el profesor como el alumno pueden compartir el mismo espacio y tiempo físico. Las nuevas ciencias aplicadas se han convertido en instrumentos pedagógicos, que constituyen el "plus del aprendiz", lo aprendido se convierte en recurso que posibilita de manera directa e indirecta tipos de exploración y aprendizaje que aportan un rendimiento cada vez mayor.

Pérez (2001) considera que la violencia es la fuerza que se ejerce en contra de unas u otras personas. Estas acciones intencionales orientadas a dañar al prójimo admiten gradación. En el ámbito escolar, encontrarnos agresiones tanto físicas como verbales a compañeros y educadores que varían en intensidad, robos, deterioro intencionado de material, insultos, burlas, amenazas, etc.

De otra parte, Restrepo (2004) asume que los maestros tienen un saber psicológico, uno antropológico, uno filosófico, diferente de los 
especialistas en estos campos del conocimiento, debido a esto, tienen la capacidad de influenciar más directamente a los estudiantes, en la educación, en la enseñanza y en la formación. Es la mirada educativa de estas ciencias sociales y humanas básicas, mirada y elaboración que constituye las ciencias de la educación.

La convivencia es un aprendizaje: se enseña y se aprende a convivir. Por ello, la convivencia es la relación entre el espacio escolar y los diversos integrantes de la comunidad educativa: estudiantes, docentes, directivos, asistentes de la educación, padres, madres, apoderados y sostenedores (Nail, Gajardo, Muñoz, 2012). Al igual que otras disciplinas científicas la investigación educativa se ha visto influenciada por las tecnologías de la información, principalmente en sus procesos de análisis. Unos profesores que se muestran muy receptivos a indagar en los principios de la interculturalidad, respetando el valor de la diferencia como referente importante en sus prácticas pedagógicas, y haciendo un análisis efectivo de las diferentes cuestiones que se ponen en juego cuando un alumno de origen inmigrante acude al centro educativo.

En el contexto de una escuela que necesita ahondar más en su capacidad inclusiva y acogedora tanto desde el punto de vista curricular como metodológico, consideramos que la formación intercultural de todos los agentes educativos es un pilar fundamental que generalmente se obvia (Cantero, 2014; Olivencia, 2012).
A partir de los aportes de Antúnez (1994), para lograr la convivencia escolar se requiere de diversos elementos, desde aquellos relacionados a la infraestructura y servicios que ofrece la institución educativa hasta la voluntad y compromiso de todos sus miembros (alumnos, alumnas, padres, familiares, docentes, directiva, personal administrativo obrero, entre otros). Además, se requiere que esto se haga de manera responsable, prestando atención a las expectativas que tienen los diferentes involucrados, incluyendo las comunidades locales y el medio ambiente (Hernández \& Jiménez, 2015), esto permitirá garantizar el equilibrio de todas las personas que forman parte de este cambio (Sisiruca \& Salazar, 2014)

Ahora bien, al analizar la experiencia de diversas instituciones educativas en su construcción de una convivencia escolar se evidencia que los "reglamentos internos para la convivencia escolar" constituyen un punto de partida para mejorar las relaciones de convivencia entre sus miembros y establecer pautas y mecanismos para la resolución de los conflictos de forma positiva.

Es así como Rodríguez (2007) considera que convivir es relacionarse unos con otros en base a ciertas relaciones sociales, en el marco de un contexto social determinado. Se debe aceptar que el hecho de que los conflictos son inseparables de la convivencia democrática no se puede pretender que la educación transcurra sin conflictos, ni pensar que estos resultaran negativos. Sin 
embargo, en las escuelas cuando un conflicto hace demasiado ruido lo que se intenta es silenciarlo bajo la creencia de que es más provechosa su desaparición ya que de esta forma se recupera la sensación de tranquilidad. La realidad es que si se tomaran los conflictos como oportunidades de aprendizaje, resultaría más sencilla su gestión, al tiempo en que aumentaría la posibilidad de resolverlo y la de poder resolverlo en el caso de que se presente un caso futuro. (Rodríguez, 2007).

En tal sentido, Graffe (2002) argumenta la importancia que tiene establecer indicadores, criterios y un sistema de información para evaluar y retroalimentar al personal encargado sobre el avance y nivel de cumplimiento de los objetivos, metas y actividades previstas, con la finalidad de mantener una constate evaluación de los resultados e impactos en término de rendimiento de alumnos y docentes. En ocasiones, se espera menos de los alumnos desde el punto de vista de su rendimiento escolar, el rendimiento de los alumnos en áreas académicas en ocasiones está estrechamente relacionado con la convivencia y forma de interactuar este en el campo de las relaciones interpersonales (Olivencia, 2009).

Considerando que la violencia ya está puesta de manifiesto, y convencidos de que la mayor parte de los espacios y tiempos que acontecen en los centros educativos son pacíficos (Grande, 2010). Lo que se pretende con este trabajo es visualizar prácticas educativas favorecedoras de una buena convivencia, realizar un análisis de las mismas y extraer algunas conclusiones sobre las condiciones y actuaciones que favorecen la mejora de la convivencia escolar.

\section{El marco de la convivencia escolar}

En los últimos años comenzaron a cuestionarse los regímenes disciplinarios escolares indiscutidos durante mucho tiempo, por su desactualización, por su rigidez burocrática, por su despersonalización, pues no reconocían a los niños, adolescentes y jóvenes, los alumnos como sujetos de derecho y responsabilidad (Ianni,2003). Con la Filosofía de los Derechos Humanos, una de las mejores herramientas para mejorar la convivencia y prevenir la violencia, tanto dentro como fuera de la escuela. Para mejorar la convivencia educativa y prevenir la violencia es necesario enseñar y aprender a resolver conflictos de forma constructiva, pensando, dialogando, negociando, a través de la mediación, de la colaboración; y creando contextos normalizados, como las asambleas de aula (Díaz, 2002)

La escuela debe fomentar una educación para la paz, formando un ciudadano autónomo y con valores cívicos, que asuma el compromiso, la responsabilidad y el derecho que tiene de vivir en una sociedad sin odios, divisiones ni violencia (Orellano,2007). Si un estudiante es fomentado bajo una educación sana, llena de compromisos y con muchos valores, posiblemente el tema de con- 
flicto escolar se vería reducido de cierta manera, de lo que podemos decir que la forma de tratar y educar a una persona depende e influye de cierta forma en el desarrollo de la personalidad de un individuo.

El término bullying, es hoy común en la literatura, sobre el tema del maltrato entre escolares, para referir la situación de violencia mantenida, mental o física, guiada por un individuo o por un grupo, dirigida contra otro individuo del grupo, quien no es capaz de defenderse. El problema, resulta preocupante, en la sociedad industrializada que parece haber alcanzado un mayor nivel de bienestar, se encuentran cada día mas agresiones y abusos en los entornos escolares, los cuales son detectados cada vez mas de manera alarmante en individuos de edades muy tempranas (Cerezo, 2009).

\section{Investigación como estrategia pedagógica apoyada en TIC}

Orientados bajo la estrategia pedagógica del Programa Ondas, la investigación busca formar el recurso humano, mediante la apropiación social del conocimiento científico y tecnológico de Colciencias, donde el eje fundamental es fomentar una cultura ciudadana en ciencia, tecnología e innovación en niños, niñas y jóvenes colombianos, reconociendo en ellos su capacidad de exploración, observación, preguntar sobre sus entornos, necesidades y problemáticas, mediante el diseño de proyectos, donde ellos organizan sus interrogantes para resolverlos desde el proceso de indagación. (Báez, 2015).

La IEP es propuesta como una metodología para desarrollar habilidades y capacidades, buscando producir, modificar, almacenar $\mathrm{y}$ compartir saberes, con formas propias de la cultura infantil y juvenil. Este tipo de pedagogía, favorece el aprendizaje por descubrimiento, debido a que los estudiantes al investigar reconocen y descubren otras realidades, se relacionan con el entorno y buscan modificarlo, establecen relaciones entre fenómenos, distinguiendo causas y consecuencias asociadas a ellos adquiriendo conocimientos con sentido práctico. (Manjarrez, 2007).

Esta tiene como finalidad instaurar bases científicas a los estudiantes en el aula de clases, esta parte de una dinámica metodológica, que busca dar respuestas a los interrogantes de los niños y niñas de la Institución y que asigna a los docentes un nuevo rol en el aula, para que, en el momento indicado, puedan ser ellos mismos quienes busquen comprender el mundo que los rodea e interpretar y describir sus problemas en un lenguaje sustentado en la ciencia. En la implementación de la IEP, se parte de la pregunta del sentido común de los niños, niñas y jóvenes para reelaborarla en la perspectiva de la educación popular, que reconoce la existencia de saberes comunes y elaborados así en la negociación con los conocimientos disciplinares, y de las maneras como se correlacionan en la negociación cultural. (Mariño, 2010). 


\section{Metodología}

El estudio se desarrolló de tipo cualitativo, desde la metodología de la investigación como estrategia pedagógica (IEP), este tipo de investigación pretende reconocer y comprender el entorno donde se encuentran los participantes teniendo como insumo sus vivencias y obteniendo una información de carácter individual de la problemática (Hernadez Sampieri, 2014)

El alcance de la investigación es descriptivo, debido a que se pretende identificar todos aquellos factores que desde la investigación van a permitir describir situaciones de interés, particularidades y tipos de personas, problemáticas, grupos o comunidades en general (Hernadez Sampieri, 2014).

\section{Participantes}

Para el estudio se tomó como población participante a (80) estudiantes distribuidos en los grados de sexto $\left(6^{\circ}\right)$ a noveno $\left(9^{\circ}\right)$ de la Institución Educativa Departamental San José, sede principal, ubicada en el municipio de Pueblo viejo Magdalena.

\section{Técnicas e Instrumentos}

Para la recolección de la información dado el tipo de investigación cualitativa se selecciona como técnica de recolección de datos la observación, y como instrumento un diario de campo, en este se registran todos aquellos datos que extraen del proceso de observar contemplando aspectos como el contexto, las posiciones y determinaciones de los participantes (Ramos, 2013)

Se implementó además los recorridos de las trayectorias de indagación, basados en la integración de la IEP apoyada en TIC, donde se establecieron cinco (5) trayectos o momentos que especifican el proceso y los fines trazados para darle solución a la pregunta problema diseñada.

\section{Procedimiento}

El estudio se desarrolló por medio de los momentos planteados por la IEP, tales como:

\section{Momento 1.}

Etapa de conformación de grupos:

Se realizó la conformación de los grupos con los participantes, donde cada uno de ellos tenía 10 integrantes teniendo un total de ocho grupos (8), a cada grupo se le asignó un tema de consulta con el que debían identificar inicialmente las fallas con relación a la convivencia escolar.

\section{Momento 2.}

Definición de líneas temáticas:

Después de haber constituido los grupos, a cada uno se les asigno un valor (honestidad, tolerancia, 
amistad, respeto, responsabilidad, amor, prudencia y gratitud) el cual debían investigar para posteriormente representar como contribuyen a la sana convivencia, y además caracterizar las técnicas de investigación implementadas.

\section{Momento 3.}

Acompañamiento para el diseño de trayectorias de indagación:

A partir de la elaboración de la actividad se identifican factores en los cuales los estudiantes presentan fallas en la convivencia escolar y además se registran comportamientos, actitudes y expresiones que acompañan a las opiniones que cada grupo plantea desde el valor humano ordenado.

\section{Momento 4.}

Acompañamiento para la producción de saber y conocimiento:

En esta etapa corresponde a que los estudiantes participantes además de identificar sus falencias desde el valor humano asignado a cada grupo perteneciente, lograron comprender la dinámica de los otros grupos y asemejar las fallas de cada uno referente a los otros valores analizados.
Momento 5.

Acompañamiento para la propagación de ciclón:

En este último momento, se realizaron obras de teatro en las que los grupos ejemplificaron las situaciones problemas y las situaciones ideales de sana convivencia, y al final de cada acto se daba por parte del grupo reflexiones acerca de su experiencia y como a través de las técnicas de investigación alcanzaron a aprender y generar nuevos saberes.

\section{Resultados}

Con la implementación del presente proyecto se logró mejorar el ambiente escolar, las relaciones interpersonales, la tolerancia, el respeto a las normas establecidas en el manual de convivencia, se minimizaron los actos de agresión física y verbal (acoso escolar), además se fortalecieron otros valores tales como, honestidad, amor, responsabilidad, prudencia y amistad.

Se afirmaron los valores de tal manera que se ven reflejados en su vida cotidiana y además en un futuro se potencializaran en sus relaciones sociales, en este sentido se logró contribuir para la formación de seres capaces de resolver conflictos en el día a día, teniendo en cuenta que los estudiantes demostraban que las fallas diarias se debían a razones como que no toleran que otros 
compañeros tengan otros puntos de vista, que consideran que los demás compañeros deben obedecer a sus peticiones, no se observaron frecuentes muestras de amabilidad; ya que la forma de pedir algo es de forma demandante y casi siempre no dan las gracias.

Al identificar las problemáticas presentadas en la comunidad estudiantil de la institución los docentes realizaron un proceso de reflexión sobre su práctica pedagógica, identificando la necesidad de integrar nuevas estrategias pedagógicas que promovieran el mejoramiento de las actitudes de los estudiantes en las aulas de clases y fuera de ellas, puesto que se observó que impartiendo el modelo tradicional de enseñanza no motiva al estudiante a cumplir con las actividades asignadas, y a implementar las estrategias de investigación que el docente trata de enseñar, en cambio cuando se desarrollaron las actividades a través de los roll plays u obras de teatro si se mostraron interesados en investigar y realizar aportes basados en la información encontrada

En este sentido los maestros investigadores proponen que para el mejoramiento de la convivencia escolar en la institución es importante utilizar las técnicas de investigación como una estrategia pedagógica, a través de la organización de equipos de trabajos liderados por un maestro y agrupados según intereses de los estudiantes, con la intención de poder entrar a fondo en temas que capten la atención de los estudiantes.
En este orden de ideas se propone realizar salidas de campo, mesas redondas y debates donde se discutan temas de interés de investigación; por ejemplo, habilidades sociales, relaciones interpersonales, manejo de conflictos y trabajo en equipo, tomándose como un ejercicio constante durante todo el año lectivo.

\section{Discusión}

La investigación como una estrategia pedagógica para fomentar la sana convivencia fue identificada por los docentes como un medio para que los estudiantes aprendieran que los valores humanos son indispensables en todos los ámbitos de la vida, y que van a contribuir de manera significativa en su desarrollo personal y social, así como lo mencionan algunos autores convivir en armonía con los demás amerita que el espacio que sea compartido represente una zona de desarrollo o progreso sin que los actos propios o ajenos originen limitaciones para el otro, es relevante tener en cuenta que el primer grupo de convivencia es la familia y por lo tanto es importante formar buenas relaciones interpersonales desde allí, para tener un desenvolvimiento optimo en otros lugares como la escuela y el trabajo (Ocampo, Briceño, Hernández \& Olano, 2010).

En este sentido la actitud frente a la creación de nuevos saberes a partir de la investigación también es de gran valor teniendo en cuenta que la convivencia está ligada firmemente a la modo de actuar de las personas y en este caso los estudiantes de- 
mostraron que tendrían una buena disposición para afrontar situaciones problemáticas que se presenten con los compañeros, entonces se atañe que la educación desde la investigación como una estrategia pedagógica va a permitir que los estudiantes aumenten su capacidad de análisis lo que va a generar una mejora gradual y posiblemente constante en cuanto a las respuestas que se den a los conflictos en los grupos a los cuales pertenezcan (hogar, escuela, barrio), ya que el proceso de enseñanza-aprendizaje comprende que la educación se dirige a mejorar la visión desde los estudiantes inicialmente y toda la comunidad educativa en general (Avendaño, Cortés \& Guerrero, 2015).

Así mismo se percibe que los estudiantes desde los grados iniciales deben ser incentivados en su proceso de desarrollo el cual no solo encierra su aprendizaje, sino también el proceso de formación integral en valores, por lo tanto es de vital relevancia que los docentes implementen a la par métodos de enseñanza contemporáneos que garanticen que el estudiante adquiera los conocimientos propios de cada grado y también refuerce los valores humanos que complementan la educación que es necesaria para su interacción en la sociedad.

Finalmente, y teniendo en cuenta lo anterior las autoras Araque \& Suárez (2017) expresan que resulta vital profundizar en la práctica de un marco legal en Colombia que oriente la acción común de leyes y que además se desarrollen estrategias de inclusión y equidad con relación a los derechos de estas comunidades.

\section{Referencias}

Antequera-Manotas, E., \& CortésPeña, O. (2016). Problems, challenges, trends and prospects for special education for talented children. The European Proceedings of Social \& Behavioural Sciences-EpSBS, 16(1), 857-861.

Antúnez, S. (1994). Claves para la organización de centros escolares. Barcelona: ICE-Horsori.

Araque, F., \& Suárez, O. (2017). Reflexiones teóricas y legales del adulto mayor y la discapacidad en Colombia. Juridicas CUC, 13(1), 97-120.

Avendaño-Villa, I., Cortés-Peña, O. F., \& Guerrero-Cuentas, H. (2015). Competencias sociales y tecnologías de la información y la comunicación como factores asociados al desempeño en estudiantes de básica primaria con experiencia de desplazamiento forzado. Diversitas, 11(1), 13 36.

Cerezo, F. (2009). Bullying: análisis de la situación en las aulas españolas. International Journal of Psychology and Psychological Therapy, 9(3). 367-378.

De La Cisterna, C. A. (2010). Manual de convivencia. Santiago de Chile: Colegio Adventista de La Cisterna.

De la Concepción, A. (2015). Concepciones sobre participación de niñas, niños y adolescentes: $\mathrm{Su}$ importancia en la construcción de la convivencia escolar. Cultura Educación y Sociedad 6(2), 9-28. 
Díaz-Aguado, M. J. (2002). Convivencia escolar y prevención de la violencia. Ministerio de Educación - Instituto de Tecnologías Educativas.

Graffe, G. J. (2002). Gestión educativa para la transformación de la escuela. Revista de Pedagogía, 23(68), 495-517.

Grande, M. J. C. (2010). Convivencia escolar. Un estudio sobre buenas prácticas. Revista de paz y conflictos, 3(1), 154-169.

Gravini, M. (2010). Estilos de aprendizaje: Un factor para tener en cuenta en la orientación profesional. Cultura Educación y Sociedad, 1(1). 75-80.

Guerrero, H. R., Crissien, T. J., \& Paniagua, R. (2017). Proyectos Educativos Institucionales Colombianos (PEI): Educación Inclusiva a través de la autoevaluación. Opción, 33(84). 218-266.

Hernández, R., Fernández, C., \& Baptista, P. (2010). Metodología de la investigación. México, D.F.: Editorial Mc Graw Hill.

Hernández, H., \& Jiménez , A. (2015). Responsabilidad social empresarial, zona urbana del distrito de barranquilla: estudio etnográfico. Económicas CUC, 36(2), 9-18.

Ianni, N. (2003). La convivencia escolar: una tarea necesaria, posible y compleja. Ciudadanía, democracia y valores en sociedades plurales, 22(2).

López, J. (2003). Compartir el mismo espacio y tiempo virtual: una propuesta de investigación para la intervención pedagógica. Revista de educación, (332), 213-231.
Nail, O., Gajardo, J., \& Muñoz, M. (2012). La técnica de análisis de incidentes críticos: Una herramienta para la reflexión sobre prácticas docentes en convivencia escolar. Psicoperspectivas, 11(2), 56-76.

Ocampo, M., Briceño, S., Hernández, M., \& Olano, M. (2010). Estrategias para mejorar la convivencia en el colegio Cristóbal Colon, Instituto Educativo Distrital. Cuadernos Hispano Americanos de Psicología, 10(2), 46-61.

Olivencia, J. (2009). Educación y conflicto en escuelas interculturales: valores y concepciones pedagógicas del profesorado sobre convivencia y diversidad cultural. Educacion y diversidad=Education and diversity: Revista inter-universitaria de investigación sobre discapacidad e interculturalidad, (3), 107-149.

Olivencia, J. (2012). La formación en educación intercultural del profesorado y la comunidad educativa. Revista Electrónica de Investigación y Docencia (REID), 2, 8-31.

Pérez, V. (2001). Convivencia escolar: problemas y soluciones. Revista complutense de educación, 12(1), 295-318.

Pinto, M. \& Misas, M. (2014). La educación inicial y la educación preescolar: Perspectivas de desarrollo en colombia y su importancia en la configuración del mundo de los niños. Cultura Educación y Sociedad 5(1), 119-140.

Restrepo, B. (2004). La investigaciónacción educativa y la construcción de saber pedagógico. Educación y educadores, (7). 
Rodríguez, R. (2007). Los Planes de Convivencia como herramientas para prevenir los conflictos escolares. Revista Mex. EBSCO, 12, 1-14.

San Martín, D. (2014). Teoría fundamentada y Atlas. TI: recursos metodológicos para la investigación educativa. Revista electrónica de investigación educativa, 16(1), 104-122.
Sisiruca, M., \& Salazar , C. (2014). Valores éticos de la responsabilidad social interna en centros de producción audiovisual. Económicas CUC, 35(1), 79-90.

Valenzuela, G., \& Rochin, L. (2013). Libertad de expresión y su impacto en la democracia. Juridicas CUC, 9(1), 89-100. 